book. It is highly recommended for all workers interested in eleft lip and palate anomalies. D. GREER WALKER

\section{PULSE RADIOLYSIS}

\section{Pulse Radiolysis}

Edited by M. Ebert, J. P. Keene, A. J. Swallow and J. H. Baxendale. (Proceedings of the International Symposium held at Manchester, April 1965.) Pp. xii +319. (London: Academic Press, Inc. (London), Ltd.; New York: Academic Press, Inc., 1965.) $70 s$.

Pulse radiolysis involves the rapid excitation of molecules by means of a microsecond burst of high-energy electrons and the observation of the resulting transient species by spectroscopic and other techniques. This symposium was the first held to discuss this technique, which is only six years old. It was organized by the Manchester group, who are leaders in the field, and this is reflected by their involvement with the majority of the twenty-two papers presented. While the symposium was described as international, in fact only four papers came from outside the United Kingdom.

The subject is still sufficiently well defined so that Dr. Keene and Dr. Baxendale could give excellent surveys of the physical and chemical background and that a complete bibliography of the technique up to the middle of 1965 could be included in the volume. The remaining papers are concerned with the pulse radiolysis of a variety of inorganic and organic liquid systems, mostly in the form of aqueous solutions. Because the technique is concerned with fundamental radiation processes, the systems studied are simple, although Dr. Braams reports studies on the reaction rate of a variety of biological molecules with the hydrated electron. The discussion following the papers is given in full.

This book is an excellent introduction to pulse radiolysis and is essential reading for all those interested in radiation chemistry, even if only to demonstrate the colossal increase in our knowledge of the primary effects of ionizing radiation which this technique has brought about.

A. KNOWLES

\section{POLYMER COMPENDIUM}

\section{Polymer Handbook}

Edited by J. Brandrup and E. H. Immergut. Pp. 1,243. (New York and London: Interscience Publishers, a Division of John Wiley and Sons, 1966.) 1478 .

THe growth of polymer science in the past two decades and the rapidly expanding literature which has resulted make the search for data on the physical properties of any polymer or polymeric system a long and tedious process. In this new polymer handbook an attempt has been made to help in the search for information needed in theoretical and experimental polymer work by collecting together in one volume, largely in tabular form, the data most useful to the polymer scientist. The contents consist only of those physical and chemical constants and parameters which are of fundamental interest to the scientist rather than data which may be useful to the polymer engineer or fabricator. The data listed are either the physical and chemical constants of the polymer molecule or constants of existing physical laws describing the propertîes and behaviour of polymers. No critical evaluation of the published values is attempted, and the numerous tables list all data found in the literature except for values which in their judgment the authors consider to be obviously erroneous.

The contents are divided into six major groups: Polymerization and synthesis. This section summarizes the constants of the polymerization process-decomposition rate of initiators, propagation and termination constants in free radical polymerization, transfer constants, reactivity ratio in copolymerization, heats and entropy of polymerization, and so on. Solid state properties, which include crystallographic data, glass transition tempera. tures, rate of crystallization and critical surface tension of polymers. Solution properties, such as viscosity, molecular weight relationships, sedimentation aad diffusion constants, second virial coefficients, polymer-solvent interaction parameters, theta solvents, dissymmetry and particle scattering factor, and so on. Physical constants of important polymers, including cellulose materials, polyethylene, polystyrene, polybutadiene, rubbers, polyacrylonitrile and poly(hexamethylene adipate). Physical constants of olegomers and physical constants of monomers and solvents. Finally, there is a large section on miscel. laneous properties such as thermal degradation, high resolution nuclear magnetic resonance, optically active polymers and radiation chemical yields. The final chapter of the handbook summarizes the trade names and practical properties of some contemporary plastic materials. An extensive subject index adds greatly to the value of the book.

As may be judged from the selection of data listed here, the book is an extremely valuable practical aid to the polymer scientist. The compilation has been a tremendous effort by a large number of distinguished workers, and the reward for their careful and painstaking work will be the appreciation of their colleagues, whose life will now be made much easier. C. E. H. BAwN

\section{ATMOSPHERIC OPTICS}

\section{Twilight}

A Study in Atmospheric Optics. By G. V. Rozenberg. Authorized translation from the Russian by Richard B. Rodman. Pp. $\mathrm{x}+358$. (New York: Plonum Press, 1966.) $\$ 20$.

THE notion of gleaning information about the structure of the atmosphere from observations of the twilight is by no means new. According to Professor Rozenberg it probably originated with the scientist Alhazen in the eleventh century. As J. V. Dave points out in the foreword, however, little progress has been achieved because of the unreliability of many of the past measurements. Only in recent years have attempts been made to adopt a standard type of sky photometer.

In spite of artificial satellites providing obvious possibilities for twilight studies, it is clear that several of the physical parameters of the high atmosphere can be more expeditiously examined by indirect ground-based methods. A vast amount of data could be gathered from a carefully chosen network of observing stations for a relatively modest expense. An indication of what might be accomplished is admirably set forth in this English translation of Rozenberg's treatise.

It is particularly useful to have readily available the Russian references to work in this field, to which the contribution of the author is considerable. Much of the text concerns ideas conceived while he was at the Atmospheric Optics Laboratory of the Institute of Atmospheric Physics, U.S.S.R. Academy of Sciences. Very recent work is mentioned in an appendix including references up to 1965. This is an indication of the rapid development of the subject, bearing in mind that a condensed version of the book was published in 1963 .

The five chapter headings range from a survey of observational results through the structure and optics of the atmosphere, followed by sections on the principles of twilight theory and the anatomy of twilight phenomena, to the inverse theory. The latter is a discussion of the problem of the interpretation and efficient organization of twilight observations. The appeal of a work of this range depends markedly on the efforts of the translator; here 